\title{
Health-related mobile apps and behaviour change
}

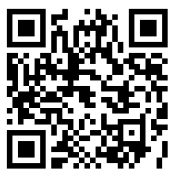

While our knowledge about physical activity and health, physical performance and the risk of injury increases in leaps and bounds, the conversion of this information into action and changed behaviour lags behind. There seems to be a sticking point which often causes a delay in new knowledge being adopted and implemented. Many examples exist, most notably, smoking. It took several decades for the prevalence of smoking to be reduced, despite an abundance of information showing the negative effects of active and passive smoking on human health. A less obvious example is the impact of physical activity, or lack of it, on health. Evidence from studies around the world show the positive effects of regular, moderate exercise, which include the impact of physical activity on performance, bone health, metabolic health, mental health and cognitive performance. Studies also show how regular exercise ameliorates some of the symptoms of ageing. For example, elderly people with a high level of physical activity are less likely to fall, which can result in an injury triggering a downward spiral in mobility and independence. It goes without saying that the reduction of falls will have a massive impact on both the well-being of the individual and the financial burden of health care costs.

Scientists have been challenged by the fact that a large portion of the population is less physically active than recommended, in spite of the overwhelming body of information on the positive benefits of physical activity. Even after much input from behavioural scientists, this change has been slow. Superficially it seems that the barriers between knowledge and behaviour need to be identified and removed. However, this is more complicated than it sounds because the barriers are dependent on and responsive to personal preference, age, cultural group and socioeconomic status.

A new wave of evidence shows that behavioural changes may be mediated through specific apps on mobile devices. Between 2013 and 2014 the global use of smart phones increased by 406 million. [1] There are now just under two billion mobile devices in use. A recent count estimated about 31000 health-related apps. ${ }^{2]}$ These apps are designed to prevent and manage chronic disease, monitor health behaviours and vitals. It is not unusual for someone in a meeting to stand up, politely commenting that the app on their smart phone has signalled that they have been seated for too long. Or for someone to comment that they need to walk during the lunch break to meet their 10000 steps goal for the day. There is compelling evidence, certainly at the anecdotal level, that these apps are having an effect on health-related behaviour. At a higher level of credibility, several published studies have utilised apps in health behaviour interventions. A systematic review of the published studies on apps used in health behaviour interventions supports the anecdotal observations. [2] Of the ten studies in the review which targeted physical activity as a primary measure, eight studies reported positive outcomes with the participants increasing their physical activity. The systematic review concluded that mobile apps might be considered an acceptable means of administering health interventions. However, more studies with larger samples and a rigorous research design are needed. Researchers are encouraged to conduct rigorous randomly controlled studies on this subject. This is an area that the South African Journal of Sports Medicine is going to actively promote in the future.

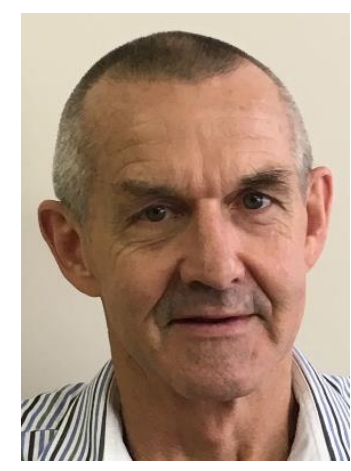

\section{Mike Lambert}

Editor-in-chief

S Afr J Sports Med 2016;28(2):35. DOI: 10.17159/2078-516X/2016/v28i2a1568

\section{References}

1. Stoyanov SR, Hides L, Kavanagh DJ, Zelenko O, Tjondronegoro D, Mani M. Mobile app rating scale: A new tool for assessing the quality of health mobile apps. JMIR Mhealth Uhealth 2015;3(1):e27 doi: 10.2196/mhealth.3422

2. Payne HE, Lister C, West JH, Bernhardt JM. Behavioral functionality of mobile apps in health interventions: A systematic review of the literature. JMIR Mhealth Uhealth 2015;3(1):e20 doi: 10.2196/mhealth.333 